\title{
New insights into the properties and interactions of carbon chains as revealed by HRTEM and DFT analysis
}

\author{
Gilberto Casillas ${ }^{1}$, Alvaro Mayoral ${ }^{2}$, Mingjie Liu ${ }^{3}$, Arturo Ponce ${ }^{1}$, Vasilii I. Artyukhov ${ }^{3}$, Boris \\ I. Yakobson ${ }^{3}$, and Miguel Jose-Yacaman ${ }^{1, *}$ \\ ${ }^{1}$ Department of Physics and Astronomy, University of Texas at San Antonio, One UTSA Circle, \\ San Antonio, Texas 78249, USA \\ ${ }^{2}$ Laboratorio de Microscopias Avanzadas (LMA), Instituto de Nanociencia de Aragon, Universidad \\ de Zaragoza, Mariano Esquillor, Edificio I+D, 50015, Zaragoza, Spain \\ ${ }^{3}$ Mechanical Engineering \& Materials Science Department, Rice University, Houston, Texas, \\ 77005
}

\begin{abstract}
Atomic carbon chains have raised interest for their possible applications as graphene interconnectors as the thinnest nanowires; however, they are hard to synthesize and subsequently to study. We present here a reproducible method to synthesize carbon chains in situ TEM.

Moreover, we present a direct observation of the bond length alternation in a pure carbon chain by aberration corrected TEM. Also, cross bonding between two carbon chains, $5 \mathrm{~nm}$ long, is observed experimentally and confirmed by DFT calculations. Finally, while free standing carbon chains were observed to be straight due to tensile loading, a carbon chain inside the walls of a carbon nanotube showed high flexibility.
\end{abstract}

\section{Introduction}

Carbon chains have recently attracted much attention since their discovery back in the 1967 [1]. Several researchers have synthesized carbon chains by chemical methods, such as functionalizing the chain ends in order to stop them from reacting with other molecules [2, 3]; however, when thinking of applications in electronic devices $(e . g$. graphene interconnections), the capping ends will change the properties of these chains, therefore a study of carbon chains in a pure carbon environment is very desirable. Troiani et al. were able to synthesize and image directly the carbon chain structure in situ in a transmission electron microscope (TEM) [4]. They achieved this by condensing the electron beam into a small area of amorphous carbon, opening holes and then thinning the bridge between them, transforming the amorphous carbon to carbon nanotubes (CNT) which would break into carbon chains [5]. Later, with the great impact that graphene produced as a possible substitute for silicon in electronic devices, two works were able to derive carbon chains from graphene sheets [6, 7]. Even though they used aberration corrected TEM (AC-TEM), which allows a resolution below $1 \AA$, they were not able to resolve the bond length in the chain.

(C) 2013 Elsevier Ltd. All rights reserved.

*Corresponding author. Tel/Fax: +1 210458 6954. miguel.yacaman@utsa.edu.

Publisher's Disclaimer: This is a PDF file of an unedited manuscript that has been accepted for publication. As a service to our customers we are providing this early version of the manuscript. The manuscript will undergo copyediting, typesetting, and review of the resulting proof before it is published in its final citable form. Please note that during the production process errors may be discovered which could affect the content, and all legal disclaimers that apply to the journal pertain. 
Since experimental manipulation of carbon chains is extremely hard, several theoretical works have been published regarding the properties of this structure; it has been predicted a Young's modulus comparable to CNTs [8, 9], spin polarized electronic transport [10], magnetic states [11], axial torsion effects [12], negative differential resistance [13], among others $[10,14-22]$.

Crystal structures involving polyynes have been studied in the literature [23-25]. More recently, a molecular dynamics study of the crystal structure of perfect carbon chains was done by Belenkov et al. [26], where they found that a crystal structure of pure carbon chains cannot exist at room temperature without accounting for cross bonding between the chains in the crystal. Other studies related to the stability of the carbon chains also showed that two carbon chains cannot form bonds easily and similar structures are quite stable chemically [27]. However, previous studies hardly revealed the polyyne structures in carbon chains nor studied how two chains interact with each other.

In the present work, we present a direct measurement of the bond length alternation in a chain from AC-TEM, which is confirmed by theoretical studies and atomic simulation, known as Peierls instability [28]. Also, we report experimental observation of cross bonding between two carbon chains by in situ TEM experiments. Density functional theory (DFT) calculations showed that carbon chains are relatively stable when two carbon chains form bonds every 9 member links, which was consistent with the experimental observations. Moreover, we present a reproducible methodology to form pure carbon chains in situ TEM by irradiation of few-layer-graphene flakes with the electron beam at room temperature. The resultant carbon chains varied in length from about $1 \mathrm{~nm}$ to $5 \mathrm{~nm}$. The dynamic process showed that carbon chains are very flexible as it confirmed that the bending stiffness is very small as in previous works.

\section{Experimental}

\subsection{TEM characterization}

Few-layer-graphene (FLG) sheets were synthesized from worm-like exfoliated graphite [29] and then drop-casted onto a lacey-carbon copper grid (Fig. S1). In situ TEM experiments were performed in a JOEL JEM-2010F equipped with a field emission gun operated at 200 $\mathrm{kV}$. The micrographs were recorded with a Fast-Scan camera with an exposure time of 0.066 s. AC-TEM experiments were performed in a FEI Titan 80-300 cubed operated at 300 $\mathrm{kV}$, equipped with a spherical aberration corrector in the lower objective lens, achieving a resolution below $0.1 \mathrm{~nm}$. All the experiments were performed at room temperature.

Once the FLG were inside the TEM the formation process was carried out as described by Caudillo et al. [5]. Briefly, holes were formed by condensing the electron beam into a small area of a few $\mathrm{nm}(\sim 2 \mathrm{~nm})$ on the FLG. Afterwards, a second hole was drilled $5 \mathrm{~nm}$ away from the first one, forming a carbon bridge between the two holes. This bridge was thinned out by focusing the beam onto it while recording the process. Due to the radiation, the carbon atoms rearrange themselves between the two holes forming carbon fibers (i.e., MWCNT) [30].

It is worth noting that if the holes were too small they would close before the thinning of the bridge. Since FLG was used as starting point, some layered structure remained after irradiating with the electron beam. If the holes were not big enough the layers would move towards the center of the holes and close them, but without reconstructing bonds. Once the holes are big enough (around $20 \mathrm{~nm}$ ) the beam was focused on the bridge area; most of the times MWCNTs were observed composing the bridge. These MWCNTs broke starting from the inner one finally leaving a single walled CNT. It is important to notice that every time an 
inner CNT broke an atomic chain was formed suggesting that they are more stable inside a CNT, which is consistent with a previous work [31].

\subsection{Modeling}

Total energy calculations were performed using the first-principles DFT with the generalized gradient approximation (GGA) of PBE with VASP [32-34]. Kinetic cutoff energy was taken to be $400 \mathrm{eV}$. All the atomic positions and lattice parameters of the structures have been optimized by minimizing the total energy, forces on atoms, as well as the stress on the structure. Structural relaxation was done until the forces acting on each atom were less than $0.01 \mathrm{eV} / \AA$. The analysis of the stability was carried out by optimizing different structures under every configuration by changing the unit cell size and relaxing the whole structure. The structure for each configuration has been chosen corresponding to the lowest energy. To remove spurious interactions between neighboring images in periodic calculations, a vacuum $12 \AA$ in all non-periodic direction was taken. A $20 \mathrm{~K}$ points mesh has been employed.

\section{Results}

\subsection{Carbon chain properties}

A variety of different phenomena were observed during the experiments. Fig. 1 shows a sequence of a typical in situ experiment of the formation of the atomic carbon chains. Fig. 1a shows an already formed chain inside a double wall CNT. As the electron beam interacts with the multi walled CNTs (MWCNT), the inner CNT shell breaks and forms another carbon chain (Fig. 1b-d), which breaks from one end and forms a ring of carbon atoms (see supplementary movie 1). Finally the outer CNT shell breaks forming a free standing chain (Fig. 1f). Interestingly, in some experiments the last CNT would break into 2 carbon chains bridging the open ends of the CNT as shown in Fig. S2.

Fig. S3 shows 6 different chains that formed during the experiments; the lengths of the formed chains varied from $1.3 \mathrm{~nm}$ to $2.24 \mathrm{~nm}$ in these cases (assuming a bond length of 0.14 $\mathrm{nm}$, they would contain 9 and $16 \mathrm{C}$ atoms). The percentage of successful formation of the chains was more than $80 \%$ for a dose of $42 \mathrm{~A} / \mathrm{cm}^{2}$. However, when a lower electron dose was applied, $5 \mathrm{~A} / \mathrm{cm}^{2}$, the ratio of success decreased to $10 \%$, obtaining smaller $\mathrm{C}$ chains (2 or 3 atoms). DFT calculations by Marques et al. [35] showed that a CNT under tension would break into a chain if it has defects present, otherwise, it will just break and close the open ends. In this sense, the energy of the electrons plays an important role since the carbon knock-on threshold is $\sim 80 \mathrm{kV}$ [36]; therefore, using energies of 200 and $300 \mathrm{kV}$, and at the same time using a high electron beam dose, the probability of creating defects in the CNTs (e. g., Stone-Wales, vacancies) increases leading to the formation of chains. This phenomenon may knock carbon atoms to the vacuum; however, the thinning process is mainly due to diffusion of the atoms away from the neck region, while the vacancies cluster to form larger holes in the structure or result in surface reconstruction of the CNTs. The formation of the atomic chains has to be a balance of vacancies creation and atoms diffusion since vacancies clustering leads to brittle fracture, while atom rearrangement leads to plastic fracture [5]. Fig. S4 shows an AC- TEM image of a carbon chain where a pentagon is observable on the right side of the chain (circled), confirming the role of the defects in the formation of the carbon chains. It is worth noting that none of the chains broke in-between, but at the junction with the nanotubes being in agreement with what Jin et al. reported [6]. While all the free standing chains are really straight due to the tensile stress they are subjected to, the one shown in Fig. 2 may have been subjected to a less tensile stress inside the CNT, allowing it to bend due to the electron beam (marked with an arrow) making an angle of $120^{\circ}$ (see supplementary movie 2). This observation is validated by the work of $\mathrm{Hu}$ 
et al. [37], where, by means of DFT calculations, they showed the strain energy even due to high bending angles of the chain is much smaller than that necessary to break a carbon bond, making it very unlikely that a carbon chain can be broken by bending.

Bond length alternation was predicted by Peierls for any atomic chain [28]. It has been proven that this dimerization actually occurs for polyyne structures [3]; however, no measurement has been done on a carbon chain in a pure carbon environment. Fig. 3 shows an AC-TEM image of a small carbon chain that formed after the breaking of a CNT. Here, it is possible to distinguish individual atoms in a short atomic chain composed of four atoms. The distance between the middle two atoms is about $1.5 \pm 0.37 \AA$, while the distance between the atoms on the right side of the chain is about $1.3 \AA \pm 0.37$. Although the experimental error is not so low, it is evident from the image that the distance between the atoms is different from one pair to the other. This confirms the dimerization caused by the Peierls instability in the atomic chain. While there is a discrepancy between the experimental bond lengths and the theoretical values, the length alternation is evident. Possible reasons for this discrepancy are experimental error, but more importantly the fact that the carbon chain may be under strain. Cretu et al. show the difference in bond length increases when the carbon chains are under tensile stress [38]. According to their results, the chain has a strain of about $10 \%$, which is not surprising since the in situ synthesis process involves applying tensile stress to the carbon nanostructures.

\subsection{Interactions between carbon chains}

Fig. 4 shows the process of formation of two carbon chains $5 \mathrm{~nm}$ long until they break (see supplementary movie 3 ). Fig. $4 \mathrm{a}$ and $\mathrm{b}$ shows the beginning of the process where there are three clearly visible chains vibrating one on top of the other. In Fig. $4 \mathrm{c}$ the three chains align one on top of the other to gain stability and grow in length. After stretching a while one of them breaks (Fig. 4d, pointed by a black arrow) leaving only two (Fig. 4e). The two remaining chains stabilize for a moment under the beam and started growing larger (Fig. 4e). This process lasted for about $2 \mathrm{~s}$ until they grew up to $5 \mathrm{~nm}$ in length (35 atoms approx. in each chain) (Fig. 4f) when one of the chains broke (Fig. 4g) causing some instabilities and finally causing the last one to break (Fig. 4h). The whole process lasted about $28.8 \mathrm{~s}$ which shows the stability of the carbon chains since they were under the electron beam irradiation all the time.

Remarkably, the last chain did break somewhere in-between, contrary to previous reports [4, 6 , 7], leaving a portion of the chain attached to the other chain while a portion of the broken chain was bent backwards (marked by an arrow in Fig. 4g). The length of the broken portion can be measured clearly by the change in contrast along the chains in Fig. $4 \mathrm{~g}$, and it is approximately $1.3 \mathrm{~nm}$, which corresponds to about 9 atoms, implying that the top and bottom portions of the chain remained bonded to the other chain; therefore, it is plausible to think that the two chains are bonded every 9 atoms. It is important to note that none of the frames in the movie showed any hexagonal or different pattern discarding the possibility of a nanoribbon being formed.

DFT calculations were performed in order to investigate how two chains interact. Although the transition from $s p$ to $s p^{2}$ hybridization for a carbon atom would decrease the energy by about $1 \mathrm{eV}$, which is the cohesive energy difference between carbon chains and graphene based on DFT, forming edges would increase the total energy at the same time. Fig. 5 shows the energy differences between two isolated carbon chains and two carbon chains forming bonds periodically (e. g., if the period is every two atoms, it forms the structure which looks like the narrowest zigzag ribbons). The ribbon with hexagonal lattice turns out to be unstable and spontaneously separates in two parallel chains. However, if the bonds formed in a dilute limit, where the effect of edge energy becomes that of bending energy, the energy of two 
carbon chains with bonds is comparable with isolated carbon chains. The minimum number of carbon atoms between two bonds at which cross-linking becomes energetically favorable is about $8(\sim 10 \AA)$, which is confirmed experimentally (Fig. 4). As the number between cross-links $\mathrm{N}$ becomes larger, exceeding the threshold number eight, the energy of structure with bond will be lower than isolated carbon chain; and the energy will approach to the

formation energy of one link. The fitting curve in Figure 5 is by function $\Delta E=A+\frac{B}{(N-C)}$ where the first term represents formation energy per link and second term represents bending energy of two chains.

\section{Conclusions}

We have shown that the in-situ TEM irradiation of FLG at 200 and $300 \mathrm{kV}$ is a reproducible method to synthesize carbon atomic chains either inside a CNT (formed by electron beam irradiation) or free standing. The formation of the chains inside CNTs walls is due to the combination of the easier diffusivity of carbon atoms inside the inner hollow [39] and the high electron dose at a high voltage. Another reason is that the displacement energy for an atom in the CNT wall gets smaller as a function of the diameter (1 to $3 \mathrm{~nm}$ ), hence, facilitating the creation of vacancies in the structure of the inner CNTs [36]. We have shown that a bundle of 3 chains can coexist without forming graphite-like ribbons. On the other hand, cross bonding between the chains was evidenced (between two chains) as one of the chains broke partially, leaving a portion with only one carbon chain. Studying the interactions between carbon chains is of crucial relevance in order to be able to use them for a real application, e. g., connectors in electronic circuit, interconnector between graphene sheets, etc.

\section{Supplementary Material}

Refer to Web version on PubMed Central for supplementary material.

\section{Acknowledgments}

The authors of this work would like to thank the NSF PREM Grant No. DMR-0934218, Title: Oxide and Metal Nanoparticles - The Interface between life sciences and physical sciences. The authors would also like to acknowledge THE WELCH FOUNDATION AGENCY PROJECT \# AX-1615, "Controlling the Shape and Particles Using Wet Chemistry Methods and Its Application to Synthesis of Hollow Bimetallic Nanostructures".

\section{References}

1. Goresy AE, Donnay G. A New Allotropic Form of Carbon from the Ries Crater. Science. 1968 Jul 26; 161(3839):363-4. [PubMed: 17776738]

2. Chalifoux WA, Tykwinski RR. Synthesis of polyynes to model the sp-carbon allotrope carbyne. Nat Chem. 2010; 2(11):967-71. [PubMed: 20966954]

3. Szafert S, Gladysz JA. Update 1 of: Carbon in One Dimension: Structural Analysis of the Higher Conjugated Polyynes. Chem Rev 2006. 2006 Nov 01; 106(11):PR1-PR33.

4. Troiani HE, Miki-Yoshida M, Camacho-Bragado GA, Marques MAL, Rubio A, Ascencio JA, et al. Direct Observation of the Mechanical Properties of Single-Walled Carbon Nanotubes and Their Junctions at the Atomic Level. Nano Lett. 2003; 3(6):751-5.

5. Caudillo R, Troiani HE, Miki-Yoshida M, Marques MAL, Rubio A, Yacaman MJ. A viable way to tailor carbon nanomaterials by irradiation-induced transformations. Radiat Phys Chem. 2005; 73(6): 334-9.

6. Jin C, Lan H, Peng L, Suenaga K, Iijima S. Deriving Carbon Atomic Chains from Graphene. Phys Rev Lett. 2009; 102(20):205501. [PubMed: 19519038] 
7. Andrey C, Jannik CM, Gerardo A-S, Ute K. From graphene constrictions to single carbon chains. New J Phys. 2009; 11(8):083019.

8. Castelli IE, Salvestrini P, Manini N. Mechanical properties of carbynes investigated by ab initio total-energy calculations. Phys Rev B. 2012; 85(21):214110.

9. Nair AK, Cranford W, Buehler MJ. The minimal nanowire: Mechanical properties of carbyne. Europhys Lett. 2011; 95(1):16002.

10. Zanolli Z, Onida G, Charlier JC. Quantum Spin Transport in Carbon Chains. ACS Nano. 2010; 4(9):5174-80. [PubMed: 20738122]

11. Xu B. Mechanical control of magnetic states of finite carbon chains encapsulated in single wall carbon nanotubes. Appl Phys Lett. 2010; 96(16):163105.

12. Ravagnan L, Manini N, Cinquanta E, Onida G, Sangalli D, Motta C, et al. Effect of Axial Torsion on sp Carbon Atomic Wires. Phys Rev Lett. 2009; 102(24):245502. [PubMed: 19659025]

13. Khoo KH, Neaton JB, Son YW, Cohen ML, Louie SG. Negative Differential Resistance in Carbon Atomic Wire-Carbon Nanotube Junctions. Nano Lett. 2008; 8(9):2900-5. [PubMed: 18710297]

14. Topsakal M, Ciraci S. Elastic and plastic deformation of graphene, silicene, and boron nitride honeycomb nanoribbons under uniaxial tension: A first-principles density-functional theory study. Phys Rev B. 2010; 81(2):024107.

15. Van Wesep RG, Chen H, Zhu W, Zhang Z. Communication: Stable carbon nanoarches in the initial stages of epitaxial growth of graphene on $\mathrm{Cu}(111)$. J Chem Phys. 2011; 134(17):171105-4. [PubMed: 21548665]

16. Erdogan E, Popov I, Rocha CG, Cuniberti G, Roche S, Seifert G. Engineering carbon chains from mechanically stretched graphene-based materials. Phys Rev B. 2011; 83(4):041401.

17. Ataca C, Ciraci S. Perpendicular growth of carbon chains on graphene from first-principles. Phys Rev B. 2011; 83(23):235417.

18. dos Santos RB, Rivelino R, Mota FdB, Gueorguiev GK. Effects of N doping on the electronic properties of a small carbon atomic chain with distinct $\mathrm{sp}^{\wedge}\{2\}$ terminations: A first-principles study. Phys Rev B. 2011; 84(7):075417.

19. Hobi E Jr, Pontes RB, Fazzio A, da Silva AJR. Formation of atomic carbon chains from graphene nanoribbons. Phys Rev B. 2010; 81(20):201406.

20. Lin ZZ, Ning J. Controlling the electronic properties of monoatomic carbon chains. Europhys Lett. 2011; 95(4):47012.

21. Shen L, Zeng M, Yang S-W, Zhang C, Wang X, Feng Y. Electron Transport Properties of Atomic Carbon Nanowires between Graphene Electrodes. J Am Chem Soc 2010. 2010 Aug 25; 132(33): 11481-6.

22. Zeng MG, Shen L, Cai YQ, Sha ZD, Feng YP. Perfect spin-filter and spin-valve in carbon atomic chains. Appl Phys Lett. 2010; 96(4):042104.

23. Mohr W, Stahl J, Hampel F, Gladysz JA. Bent and Stretched but Not Yet to the Breaking Point: C8-C16 sp Carbon Chains That Span Two Platinum Atoms and the First Structurally Characterized 1,3,5,7,9,11,13,15-Octayne. Inorg Chem. 2001 Jul 01; 40(14):3263-4. [PubMed: 11421667]

24. Sorokin PB, Lee H, Antipina LY, Singh AK, Yakobson BI. Calcium-Decorated Carbyne Networks as Hydrogen Storage Media. Nano Lett 2011. 2011 Jul 13; 11(7):2660-5.

25. Eisler S, Slepkov AD, Elliott E, Luu T, McDonald R, Hegmann FA, et al. Polyynes as a Model for Carbyne: Synthesis, Physical Properties, and Nonlinear Optical Response. J Am Chem Soc 2005. 2005 Mar 01; 127(8):2666-76.

26. Belenkov E, Mavrinsky V. Crystal structure of a perfect carbyne. Crystallgraph Rep. 2008; 53(1): 83-7.

27. Casari CS, Li Bassi A, Ravagnan L, Siviero F, Lenardi C, Piseri P, et al. Chemical and thermal stability of carbyne-like structures in cluster-assembled carbon films. Phys Rev B. 2004; 69(7): 075422 .

28. Kennedy T, Lieb EH. Proof of the Peierls instability in one dimension. Phys Rev Lett. 1987; 59(12):1309-12. [PubMed: 10035199] 
29. Gu W, Zhang W, Li X, Zhu H, Wei J, Li Z, et al. Graphene sheets from worm-like exfoliated graphite. J Mater Chem. 2009; 19(21):3367-9.

30. Florian B. Irradiation effects in carbon nanostructures. Rep Prog Phys. 1999; 62(8):1181.

31. Wang Z, Ke X, Zhu Z, Zhang F, Ruan M, Yang J. Carbon-atom chain formation in the core of nanotubes. Phys Rev B. 2000; 61(4):R2472.

32. Kresse G, Hafner J. Ab initio molecular dynamics for liquid metals. Phys Rev B. 1993; 47(1):55861.

33. Kresse G, Furthmüller J. Efficient iterative schemes for ab initio total-energy calculations using a plane-wave basis set. Phys Rev B. 1996; 54(16):11169-86.

34. Perdew JP, Burke K, Ernzerhof M. Generalized Gradient Approximation Made Simple. Phys Rev Lett. 1996; 77(18):3865-8. [PubMed: 10062328]

35. Marques MAL, Troiani HE, Miki-Yoshida M, Jose-Yacaman M, Rubio A. On the Breaking of Carbon Nanotubes under Tension. Nano Lett. 2004; 4(5):811-5.

36. Krasheninnikov AV, Banhart F, Li JX, Foster AS, Nieminen RM. Stability of carbon nanotubes under electron irradiation: Role of tube diameter and chirality. Phys Rev B. 2005; 72(12):125428.

37. Hu YH. Bending Effect of sp-Hybridized Carbon (Carbyne) Chains on Their Structures and Properties. J Phys Chem C. 2011 Feb 10; 115(5):1843-50.

38. Cretu O, Botello-Mendez AR, Janowska I, Pham-Huu C, Charlier JC, Banhart F. Electrical conductivity measured in atomic carbon chains. 2013 arXiv:1302.5207.

39. Krasheninnikov AV, Nordlund K, Lehtinen PO, Foster AS, Ayuela A, Nieminen RM. Adsorption and migration of carbon adatoms on carbon nanotubes: Density-functional ab initio and tightbinding studies. Phys Rev B. 2004; 69(7):073402. 

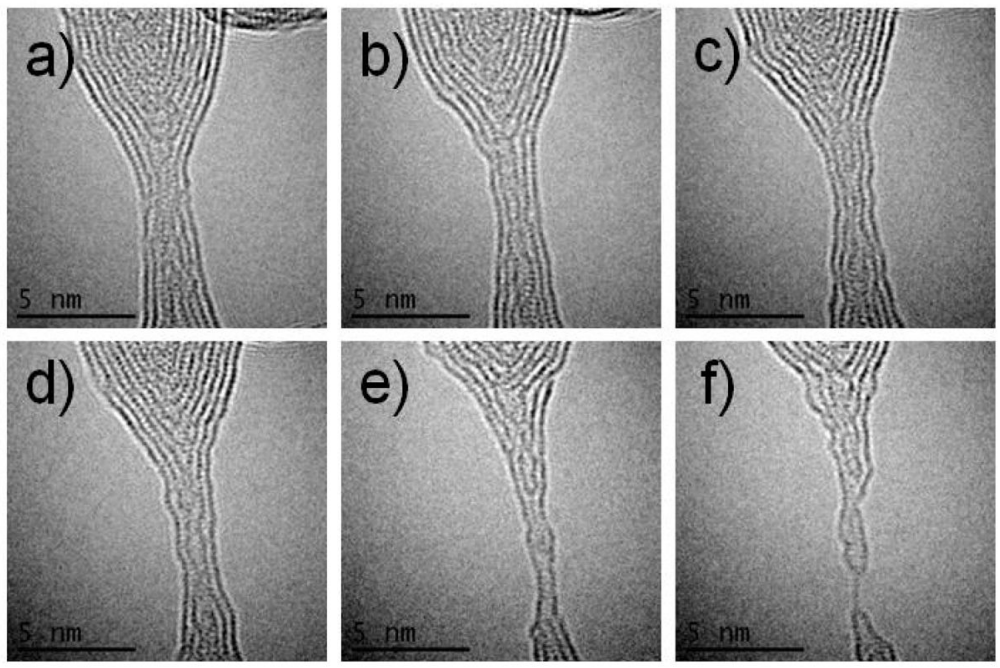

Fig. 1.

Sequence of the experiment performed in HRTEM. a) A MWCNT is formed due to the electron irradiation. b) The diameter of the MWCNT is reduced considerably. c) The number of walls starts to decrease at two. d) A double wall CNT is clearly formed. e) The inner CNT breaks and a SWCNT remains. f) The SWCNT breaks and forms the atomic carbon chain. 


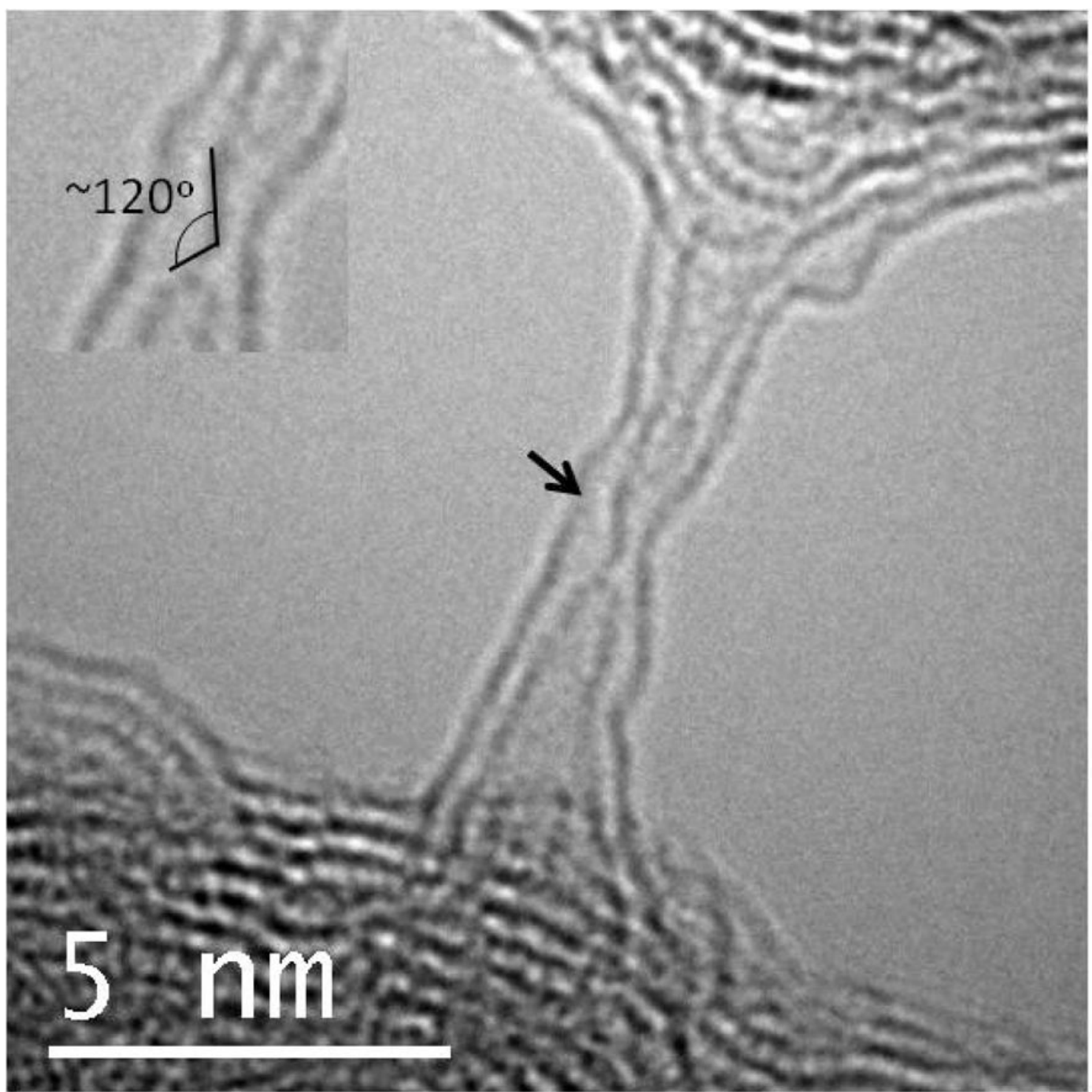

Fig. 2.

HRTEM image of a bent carbon chain inside a CNT. Inset shows a magnified image of the chain where the $120^{\circ}$ angle formed by the chain is measured 


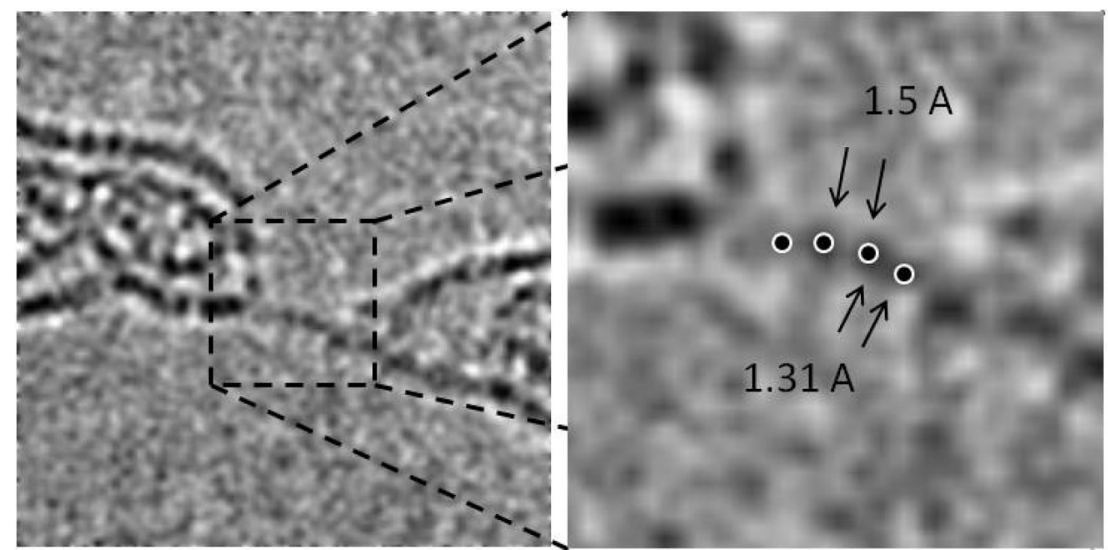

Fig. 3.

AC-HRTEM images of a carbon chain formed by 4 atoms where the bond length alternation is observed. 

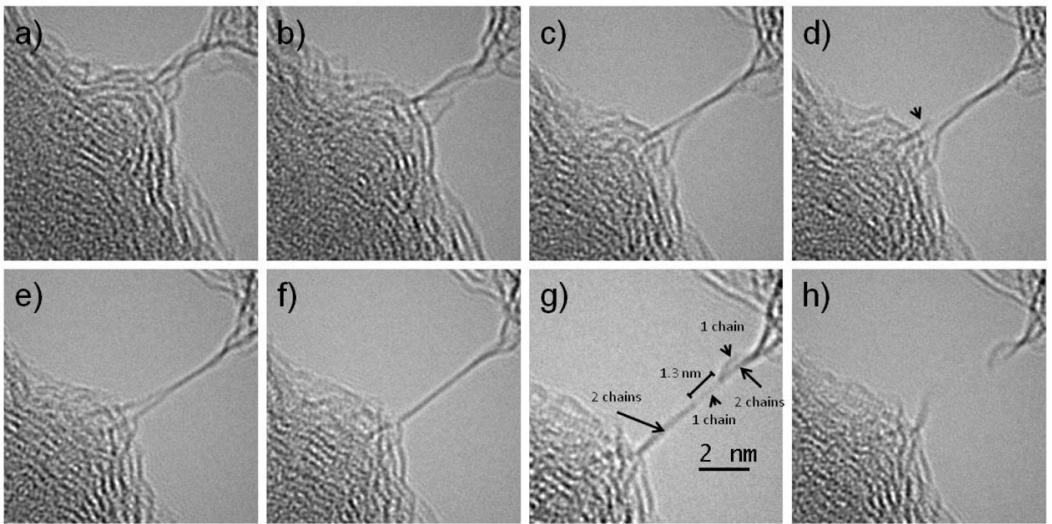

Fig. 4.

HRTEM images of the formation of three chains. From a-d) it is possible to discern three carbon chains. In d) one of the chains breaks (marked by an arrow), leaving only two long chains. In f) the two chains grow up to $5 \mathrm{~nm}$. In g) one of the chains breaks in the middle leaving a section with only one chain, shown by a decrease in contrast. h) Both chains finally break. 


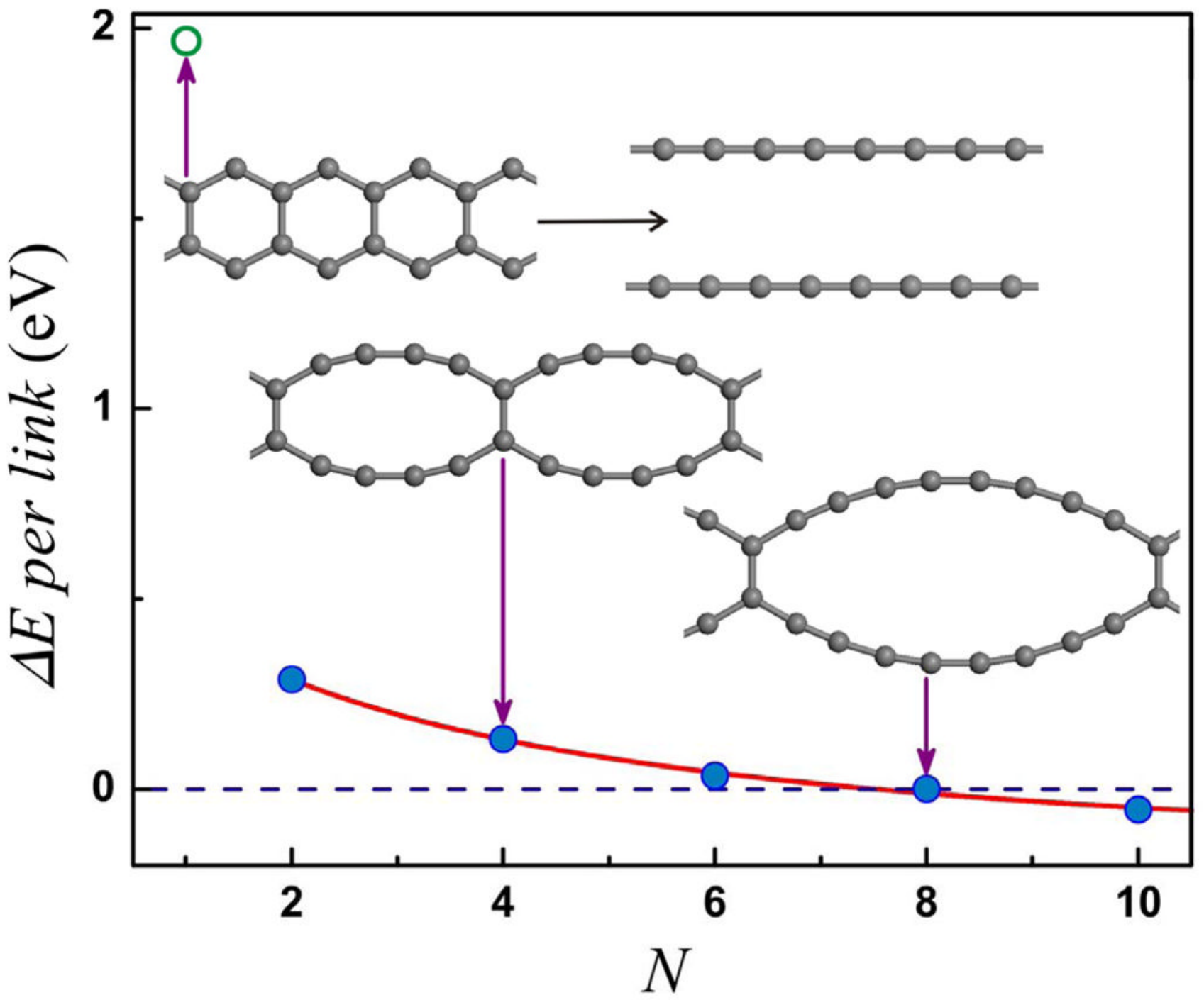

Fig. 5.

The energy difference $\Delta \mathrm{E}$ per link between cross-link structures and an isolated carbon chain calculated by DFT. Each configuration is calculated with a periodic boundary condition in one direction. $\mathrm{N}$ is the number of atom between links. The insets denote the relaxed structures of the corresponding data points. The dashed line $(\Delta \mathrm{E}=0)$ is the reference energy of an isolated carbon chain. The hexagonal nanoribbon structure is unstable. $\mathrm{N}=8$ is the threshold for two chains forming cross-links. When $\mathrm{N}$ increases, the energy will approach to the formation energy for one link. 